\title{
Positive Periodic Solutions of Cooperative Systems with Delays and Feedback Controls
}

\author{
Tursuneli Niyaz ${ }^{1}$ and Ahmadjan Muhammadhaji ${ }^{2}$ \\ ${ }^{1}$ College of Applied Mathematics, Xinjiang University of Finance and Economics, Ürümqi 830012, China \\ ${ }^{2}$ College of Mathematical Sciences, Xinjiang Normal University, Urumqi 830054, China
}

Correspondence should be addressed to Ahmadjan Muhammadhaji; ahmadjanm@gmail.com

Received 23 April 2013; Accepted 13 May 2013

Academic Editor: Norio Yoshida

Copyright (C) 2013 T. Niyaz and A. Muhammadhaji. This is an open access article distributed under the Creative Commons Attribution License, which permits unrestricted use, distribution, and reproduction in any medium, provided the original work is properly cited.

\begin{abstract}
This paper studies a class of periodic $n$ species cooperative Lotka-Volterra systems with continuous time delays and feedback controls. Based on the continuation theorem of the coincidence degree theory developed by Gaines and Mawhin, some new sufficient conditions on the existence of positive periodic solutions are established.
\end{abstract}

\section{Introduction}

Mathematical ecological system has become one of the most important topics in the study of modern applied mathematics. Its dynamical behavior includes persistence, permanence and extinction of species, global stability of systems, the existence of positive periodic solutions, positive almost periodic solutions, and strictly positive solutions. The existence of positive periodic solutions has already become one of the most interesting subjects for scholars. In the recent years, the application of fixed-point theorems to the existence of positive periodic solutions in mathematical ecology has been studied extensively, for example, Brouwer's fixed point theorem [1-4], Schauder's fixed-point theorem [5-8], Krasnoselskii's fixed-point theorem [9-14], Horn's fixed-point theorem [15, 16], and Mawhins continuation theorem [17-36], and so forth. In particular, Mawhins continuation theorem is a powerful tool for studying the existence of periodic solutions of periodic high-dimensional time-delayed problems. When dealing with a time-delayed problem, it is very convenient and the result is relatively simple [30]. Recently, a considerable number of mathematical models with delays have been proposed in the study of population dynamics. One of the most celebrated models for population is the LotkaVolterra system. Subsequently, a lot of the literature related to the study of the existence of positive periodic solutions for various Lotka-Volterra-type population dynamical systems with delays by using the method of continuation theorem was published and extensive research results were obtained [17-21, 24-34].

On the other hand, in some situations, people may wish to change the position of the existing periodic solution but to keep its stability. This is of significance in the control of ecology balance. One of the methods for the realization of it is to alter the system structurally by introducing some feedback control variables so as to get a population stabilizing at another periodic solution. The realization of the feedback control mechanism might be implemented by means of some biological control scheme or by harvesting procedure [21]. In fact, during the last decade, the existence of positive periodic solutions for the population dynamics with feedback control has been studied extensively [8,14, 17-21, 24, 29]. To the best of our knowledge, studies on the existence of positive periodic solutions for cooperative systems with delays and feedback controls are fairly rare.

In [21], the authors studied the following neutral LotkaVolterra system with feedback controls:

$$
\begin{aligned}
\dot{y}_{i}(t)=y_{i}(t)\left[r_{i}(t)-\sum_{j=1}^{n} a_{i j} y_{j}(t)\right. & \\
& \quad-\sum_{j=1}^{n} b_{i j} y_{j}\left(t-\tau_{i j}(t)\right)-\sum_{j=1}^{n} c_{i j} \dot{y}_{j}\left(t-\gamma_{i j}(t)\right)
\end{aligned}
$$




$$
\begin{gathered}
\left.-f_{i}(t) u_{i}(t)-e_{i}(t) u_{i}\left(t-\sigma_{i}(t)\right)\right] \\
\dot{u}_{i}(t)=-\alpha_{i}(t) u_{i}(t)+\beta_{i}(t) y_{i}(t) \\
+\gamma_{i}(t) y_{i}(t)\left(t-\delta_{i}(t)\right), \quad i=1,2, \ldots, n .
\end{gathered}
$$

By using Mawhin's continuation theorem, the sufficient conditions on the existence of positive periodic solutions are established. In [24], the authors considered the following delay differential system with feedback control:

$$
\begin{gathered}
\frac{d x}{d t}=F\left(t, x\left(t-\tau_{1}(t)\right), \ldots, x\left(t-\tau_{n}(t)\right), u(t-\delta(t))\right) \\
\frac{d u}{d t}=-\eta(t)+a(t) x(t-\sigma(t)) .
\end{gathered}
$$

A set of natural and easily verifiable sufficient conditions of the existence of positive periodic solutions are established, by using Mawhin's continuation theorem. In [29], the authors considered the following single-species periodic logistic systems with feedback regulation and infinite distributed delay:

$$
\begin{aligned}
& \dot{N}(t)= r(t) N(t) \\
& \times\left(1-\frac{1}{K(t)} \int_{0}^{\infty} H(s) N(t-s) d s-c(t) u(t)\right) \\
& \dot{u}(t)=-a(t) u(t)+b(t) \int_{0}^{\infty} H(s) N^{2}(t-s) d s .
\end{aligned}
$$

The sufficient conditions for the existence of positive periodic solutions are established, based on Mawhin's continuation theorem.

Motivated by the above works, in this paper, we investigate the following $n$ species periodic Lotka-Volterra-type cooperative systems with continuous time delays and feedback controls:

$$
\begin{aligned}
\dot{x}_{i}(t)= & x_{i}(t)\left[r_{i}(t)-\sum_{l=1}^{m} a_{i i l}(t) x_{i}\left(t-\tau_{i i l}(t)\right)\right. \\
& \left.+\sum_{j \neq i}^{n} \sum_{l=1}^{m} a_{i j l}(t) x_{j}\left(t-\tau_{i j l}(t)\right)\right] \\
& -d_{i}(t) u_{i}(t)-e_{i}(t) u_{i}\left(t-\varepsilon_{i}(t)\right) \\
\dot{u}_{i}(t)= & -b_{i}(t) u_{i}(t)+\beta_{i}(t) x_{i}(t) \\
& +\gamma_{i}(t) x_{i}\left(t-\sigma_{i}(t)\right), \quad i=1,2, \ldots, n .
\end{aligned}
$$

By using the technique of coincidence degree developed by Gaines and Mawhin in [36], we will establish some new sufficient conditions which guarantee that the system has at least one positive periodic solution.

\section{Preliminaries}

In system (4), we have that $x_{i}(t)(i=1,2, \ldots n)$ represent the density of $n$ cooperative species $x_{i}(i=1,2, \ldots n)$ at time $t$, respectively; $r_{i}(t)(i=1,2, \ldots n)$ represent the intrinsic growth rate of species $x_{i}(i=1,2, \ldots n)$ at time $t$, respectively; $a_{i i l}(t)(i=1,2, \ldots, n, l=1,2, \ldots, m)$ represent the intrapatch restriction density of species $x_{i}(i=1,2, \ldots n)$ at time $t$, respectively; $a_{i j l}(t)(l=1,2, \ldots, m, i \neq j, i, j=$ $1,2, \ldots, n)$ represent the cooperative coefficients between $n$ species $x_{i}(i=1,2, \ldots n)$ at time $t$, respectively. $u_{i}(t)(i=$ $1,2, \ldots n)$ represent the indirect feedback control variables [21] at time $t$, respectively. $\beta_{i}(t), e_{i}(t), b_{i}(t), d_{i}(t)$, and $\gamma_{i}(t)(i=$ $1,2, \ldots n)$ represent the feedback control coefficients at time $t$, respectively. In this paper, we always assume that

(H1) $\tau_{i j l}(t)(l=1,2, \ldots, m, i, j=1,2, \ldots, n), \sigma_{i}(t), \varepsilon_{i}(t), r_{i}$ $(t)(i=1,2, \ldots n)$ are continuous $\omega$-periodic functions with $\tau_{i j l}^{\prime}(t)<1$ and $\int_{0}^{\omega} r_{i}(t) d t>0 . a_{i j l}(t)(i, j=$ $1,2, l=1,2, \ldots, m), \beta_{i}(t), e_{i}(t), b_{i}(t), d_{i}(t)$, and $\gamma_{i}(t)(i=1,2, \ldots n)$ are continuous, positive $\omega$ periodic functions.

From the viewpoint of mathematical biology, in this paper for system (4) we only consider the solution with the following initial conditions:

$$
\begin{aligned}
& x_{i}(t)=\phi_{i}(t), \quad \forall t \in[-\sigma, 0], i=1,2, \ldots n, \\
& u_{i}(t)=\psi_{i}(t), \quad \forall t \in[-\sigma, 0], i=1,2, \ldots n
\end{aligned}
$$

where $\phi_{i}(t), \psi_{i}(t)(i=1,2, \ldots n)$ are nonnegative continuous functions defined on $[-\sigma, 0]$ satisfying $\phi_{i}(0)>0, \psi_{i}(0)>$ $0(i=1,2, \ldots n)$ with $\sigma=\max _{t \in[0, \omega]}\left\{\tau_{i j l}(t), \sigma_{i}(t), \varepsilon_{i}(t)(i, j=\right.$ $1,2, l=1,2, \ldots, m)\}$.

In this paper, for any $\omega$-periodic continuous function $f(t)$ we denote

$$
\begin{gathered}
f^{L}=\min _{t \in[0, \omega]} f(t), \quad f^{M}=\max _{t \in[0, \omega]} f(t), \\
\bar{f}=\frac{1}{\omega} \int_{0}^{\omega} f(t) d t .
\end{gathered}
$$

In order to obtain the existence of positive $\omega$-periodic solutions of system (4), we will use the continuation theorem developed by Gaines and Mawhin in [36]. For the reader's convenience, we will introduce the continuation theorem in the following.

Let $X$ and $Z$ be two normed vector spaces. Let $L$ : Dom $L \subset X \rightarrow Z$ be a linear operator and let $N: X \rightarrow Z$ be a continuous operator. The operator $L$ is called a Fredholm operator of index zero, if $\operatorname{dimKer} L=\operatorname{codim} \operatorname{Im} L<\infty$ and $\operatorname{Im} L$ is a closed set in $Z$. If $L$ is a Fredholm operator of index zero, then there exist continuous projectors $P: X \rightarrow X$ and $Q: Z \rightarrow Z$ such that $\operatorname{Im} P=\operatorname{Ker} L$ and $\operatorname{Im} L=$ $\operatorname{Ker} Q=\operatorname{Im}(I-Q)$. It follows that $L \mid \operatorname{Dom} L \cap \operatorname{Ker} P$ : Dom $L \cap \operatorname{Ker} P \rightarrow \operatorname{Im} L$ is invertible and its inverse is denoted by $K_{P}$; denote by $J: \operatorname{Im} Q \rightarrow \operatorname{Ker} L$ an isomorphism of $\operatorname{Im} Q$ onto $\operatorname{Ker} L$. Let $\Omega$ be a bounded open subset of $X$; we say that the operator $N$ is $L$-compact on $\bar{\Omega}$, where $\bar{\Omega}$ denotes 
the closure of $\Omega$ in $X$, if $Q N(\bar{\Omega})$ is bounded and $K_{P}(I-Q) N$ : $\bar{\Omega} \rightarrow X$ is compact.

Lemma 1 (see [35]). Suppose $\tau \in C^{1}(R, R)$ with $\tau(t+\omega) \equiv \tau(t)$ and $\tau^{\prime}(t)<1, \forall t \in[0, \omega]$. Then the function $t-\tau(t)$ has a unique inverse function $\mu(t)$ satisfying $\mu \in C(R, R), \mu(u+\omega)=$ $\mu(u)+\omega, \forall u \in R$.

Lemma 2 (see [36]). Let $L$ be a Fredholm operator of index zero and let $N$ be L-compact on $\bar{\Omega}$. If

(a) for each $\lambda \in(0,1)$ and $x \in \partial \Omega \cap \operatorname{Dom} L, L x \neq \lambda N x$;

(b) for each $x \in \partial \Omega \cap \operatorname{Ker} L, Q N x \neq 0$;

(c) $\operatorname{deg}\{J Q N, \Omega \cap \operatorname{Ker} L, 0\} \neq 0$,

then the operator equation $L x=N x$ has at least one solution lying in $\operatorname{Dom} L \cap \bar{\Omega}$.

\section{Main Results}

In order to obtain the existence of positive periodic solutions of system (4), firstly, we introduce the following lemma.

Lemma 3. Suppose that $\left(x_{1}^{*}(t), x_{2}^{*}(t), \ldots, x_{n}^{*}(t), u_{1}^{*}(t)\right.$, $\left.u_{2}^{*}(t), \ldots, u_{n}^{*}(t)\right)$ is an $\omega$-periodic solution of (4) and (5); then $\left(x_{1}^{*}(t), x_{2}^{*}(t), \ldots, x_{n}^{*}(t), u_{1}^{*}(t), u_{2}^{*}(t), \ldots, u_{n}^{*}(t)\right)$ satisfies the system

$$
\begin{aligned}
\dot{x}_{i}(t)= & x_{i}(t)\left(r_{i}(t)-\sum_{j=1}^{2}(-1)^{i+j} \sum_{l=1}^{m} a_{i j l}(t) x_{j}\left(t-\tau_{i j l}(t)\right)\right) \\
& -d_{i}(t) u_{i}(t)-e_{i}(t) u_{i}\left(t-\varepsilon_{i}(t)\right), \\
u_{i}(t)= & \int_{t}^{t+\omega}\left[\beta_{i}(s) x_{i}(s)+\gamma_{i}(s) x_{i}\left(s-\sigma_{i}(s)\right)\right] G_{i}(t, s) d s,
\end{aligned}
$$$$
i=1,2, \ldots, n,
$$

where

$$
G_{i}(t, s)=\frac{\exp \left\{\int_{t}^{s} b_{i}(\theta) d \theta\right\}}{\exp \left\{\int_{0}^{\omega} b_{i}(\theta) d \theta\right\}-1}, \quad i=1,2, \ldots, n .
$$

The converse is also true.

Proof. By (4), (5), and the variation constants formula in ordinary differential equations, we have

$$
\begin{aligned}
u_{i}(t)= & \left(\int_{0}^{t}\left(\beta_{i}(s) x_{i}(s)+\gamma_{i}(s) x_{i}\left(s-\sigma_{i}(s)\right)\right)\right. \\
& \left.\times \exp \left\{\int_{0}^{s} b_{i}(\theta) d \theta\right\} d s+\psi_{i}(0)\right) \\
& \times \exp \left\{-\int_{0}^{t} b_{i}(\theta) d \theta\right\}, \quad i=1,2, \ldots, n .
\end{aligned}
$$

From (9), we obtain

$$
\begin{aligned}
u_{i}(t+\omega)= & \left(\int_{0}^{t+\omega}\left(\beta_{i}(s) x_{i}^{*}(s)+\gamma_{i}(s) x_{i}^{*}\left(s-\sigma_{i}(s)\right)\right)\right. \\
& \left.\times \exp \left\{\int_{0}^{s} b_{i}(\theta) d \theta\right\} d s+\psi_{i}(0)\right) \\
& \times \exp \left\{-\int_{0}^{t+\omega} b_{i}(\theta) d \theta\right\}, \quad i=1,2, \ldots, n, \\
u_{i}^{*}(t)= & \left(\int_{0}^{t}\left(\beta_{i}(s) x_{i}^{*}(s)+\gamma_{i}(s) x_{i}^{*}\left(s-\sigma_{i}(s)\right)\right)\right. \\
& \left.\times \exp \left\{\int_{0}^{s} b_{i}(\theta) d \theta\right\} d s+\psi_{i}(0)\right) \\
& \times \exp \left\{-\int_{0}^{t} b_{i}(\theta) d \theta\right\}, \quad i=1,2, \ldots, n .
\end{aligned}
$$

Considering that $\left(x_{1}^{*}(t), x_{2}^{*}(t), \ldots, x_{n}^{*}(t), u_{1}^{*}(t), u_{2}^{*}(t), \ldots\right.$, $\left.u_{n}^{*}(t)\right)$ is an $\omega$-periodic solution of system (4) and (5), we obtain

$$
\begin{aligned}
& \left(\int_{0}^{t}\left(\beta_{i}(s) x_{i}^{*}(s)+\gamma_{i}(s) x_{i}^{*}\left(s-\sigma_{i}(s)\right)\right)\right. \\
& \left.\quad \times \exp \left\{\int_{0}^{s} b_{i}(\theta) d \theta\right\} d s+\psi_{i}(0)\right) \exp \left\{-\int_{0}^{t} b_{i}(\theta) d \theta\right\} \\
& =\left(\int_{0}^{t+\omega}\left(\beta_{i}(s) x_{i}^{*}(s)+\gamma_{i}(s) x_{i}^{*}\left(s-\sigma_{i}(s)\right)\right)\right. \\
& \left.\quad \times \exp \left\{\int_{0}^{s} b_{i}(\theta) d \theta\right\} d s+\psi_{i}(0)\right) \\
& \quad \times \exp \left\{-\int_{0}^{t+\omega} b_{i}(\theta) d \theta\right\}, \quad i=1,2, \ldots, n .
\end{aligned}
$$

Then

$$
\begin{aligned}
& \left(\int_{0}^{t}\left(\beta_{i}(s) x_{i}^{*}(s)+\gamma_{i}(s) x_{i}^{*}\left(s-\sigma_{i}(s)\right)\right)\right. \\
& \left.\quad \times \exp \left\{\int_{0}^{s} b_{i}(\theta) d \theta\right\} d s+\psi_{i}(0)\right) \exp \left\{\int_{0}^{\omega} b_{i}(\theta) d \theta\right\} \\
& =\left(\int_{0}^{t+\omega}\left(\beta_{i}(s) x_{i}^{*}(s)+\gamma_{i}(s) x_{i}^{*}\left(s-\sigma_{i}(s)\right)\right)\right. \\
& \left.\quad \times \exp \left\{\int_{0}^{s} b_{i}(\theta) d \theta\right\} d s+\psi_{i}(0)\right) \\
& =\int_{0}^{t}\left(\beta_{i}(s) x_{i}^{*}(s)+\gamma_{i}(s) x_{i}^{*}\left(s-\sigma_{i}(s)\right)\right) \\
& \quad \times \exp \left\{\int_{0}^{s} b_{i}(\theta) d \theta\right\} d s+\psi_{i}(0)
\end{aligned}
$$




$$
\begin{aligned}
& +\int_{t}^{t+\omega}\left(\beta_{i}(s) x_{i}^{*}(s)+\gamma_{i}(s) x_{i}^{*}\left(s-\sigma_{i}(s)\right)\right) \\
& \quad \times \exp \left\{\int_{0}^{s} b_{i}(\theta) d \theta\right\} d s, \quad i=1,2, \ldots, n,
\end{aligned}
$$

which implies

$$
\begin{aligned}
& \left(\int_{0}^{t}\left(\beta_{i}(s) x_{i}^{*}(s)+\gamma_{i}(s) x_{i}^{*}\left(s-\sigma_{i}(s)\right)\right)\right. \\
& \left.\quad \times \exp \left\{\int_{0}^{s} b_{i}(\theta) d \theta\right\} d s+\psi_{i}(0)\right)\left(e^{\bar{b} \omega}-1\right) \\
& =\int_{t}^{t+\omega}\left(\beta_{i}(s) x_{i}^{*}(s)+\gamma_{i}(s) x_{i}^{*}\left(s-\sigma_{i}(s)\right)\right) \\
& \quad \times \exp \left\{\int_{0}^{s} b_{i}(\theta) d \theta\right\} d s, \quad i=1,2, \ldots, n .
\end{aligned}
$$

That is,

$$
\begin{aligned}
& \int_{0}^{t}\left(\beta_{i}(s) x_{i}^{*}(s)+\gamma_{i}(i) x_{i}^{*}\left(s-\sigma_{i}(s)\right)\right) \\
& \quad \times \exp \left\{\int_{0}^{s} b_{i}(\theta) d \theta\right\} d s+\psi_{i}(0) \\
& =u_{i}^{*}(t) \exp \left\{\int_{0}^{t} b_{i}(\theta) d \theta\right\} \\
& =\frac{1}{e^{\bar{b} \omega}-1} \int_{t}^{t+\omega}\left(\beta_{i}(s) x_{i}^{*}(s)+\gamma_{i}(s) x_{i}^{*}\left(s-\sigma_{i}(s)\right)\right) \\
& \quad \times \exp \left\{\int_{0}^{s} b_{i}(\theta) d \theta\right\} d s, \quad i=1,2, \ldots, n .
\end{aligned}
$$

Hence

$$
\begin{aligned}
u_{i}^{*}(t)=\int_{t}^{t+\omega} & \left(\beta_{i}(s) x_{i}^{*}(s)+\gamma_{i}(s) x_{i}^{*}\left(s-\sigma_{i}(s)\right)\right) \\
& \times \frac{\exp \left\{\int_{t}^{s} b_{i}(\theta) d \theta\right\}}{\exp \left\{\int_{0}^{\omega} b_{i}(\theta) d \theta\right\}-1} d s, \quad i=1,2, \ldots, n .
\end{aligned}
$$

On the other hand, assume that $\left(x_{1}^{*}(t), x_{2}^{*}(t), \ldots, x_{n}^{*}(t), u_{1}^{*}(t)\right.$, $\left.u_{2}^{*}(t), \ldots, u_{n}^{*}(t)\right)$ is an $\omega$-periodic solution of system $(7)$, then

$$
\begin{aligned}
u_{i}^{*}(t)=\int_{t}^{t+\omega} & \left(\beta_{i}(s) x_{i}^{*}(s)+\gamma_{i}(s) x_{i}^{*}\left(s-\sigma_{i}(s)\right)\right) \\
& \times \frac{\exp \left\{\int_{t}^{s} b_{i}(\theta) d \theta\right\}}{\exp \left\{\int_{0}^{\omega} b_{i}(\theta) d \theta\right\}-1} d s, \quad i=1,2, \ldots, n .
\end{aligned}
$$

By a direct calculation, we have

$$
\begin{aligned}
& \frac{d u^{*}(t)}{d t} \\
& =-b_{i}(t) \int_{t}^{t+\omega}\left(\beta_{i}(s) x_{i}^{*}(s)+\gamma_{i}(s) x_{i}^{*}\left(s-\sigma_{i}(s)\right)\right) \\
& \quad \times \frac{\exp \left\{\int_{t}^{s} b_{i}(\theta) d \theta\right\}}{\exp \left\{\int_{0}^{\omega} b_{i}(\theta) d \theta\right\}-1} d s \\
& +\left(\beta_{i}(t+\omega) x_{i}^{*}(t+\omega)\right. \\
& \left.\quad+\gamma_{i}(t+\omega) x_{i}^{*}\left(t+\omega-\sigma_{i}(t+\omega)\right)\right) \\
& \times \frac{\exp \left\{\int_{t}^{t+\omega} b_{i}(\theta) d \theta\right\}}{\exp \left\{\int_{0}^{\omega} b_{i}(\theta) d \theta\right\}-1} \\
& -\left(\beta_{i}(t) x_{i}^{*}(t)+\gamma_{i}(t) x_{i}^{*}\left(t-\sigma_{i}(t)\right)\right) \\
& \times \frac{\exp \left\{\int_{0}^{\omega} b_{i}(\theta) d \theta\right\}-1}{=-b_{i}(t) u_{i}(t)+\beta_{i}(t) x_{i}^{*}(t)+\gamma_{i}(t) x_{i}^{*}\left(t-\sigma_{i}(t)\right)},
\end{aligned}
$$

This completes the proof.

It is easy to see that system (7) is equivalent to the following system:

$$
\begin{gathered}
\dot{x}_{i}(t)=x_{i}(t)\left(r_{i}(t)-\sum_{j=1}^{2}(-1)^{i+j} \sum_{l=1}^{m} a_{i j l}(t) x_{j}\left(t-\tau_{i j l}(t)\right)\right) \\
-d_{i}(t) u_{i}(t)-e_{i}(t) u_{i}\left(t-\varepsilon_{i}(t)\right), \\
u_{i}(t)=\int_{t}^{t+\omega} K\left(x_{i}\right) G_{i}(t, s) d s, \quad i=1,2, \ldots, n,
\end{gathered}
$$

where

$$
\begin{array}{r}
G_{i}(t, s)=\frac{\exp \left\{\int_{t}^{s} b_{i}(\theta) d \theta\right\}}{\exp \left\{\int_{0}^{\omega} b_{i}(\theta) d \theta\right\}-1}, \\
K\left(x_{i}\right)=\beta_{i}(s) x_{i}(s)+\gamma_{i}(s) x_{i}\left(s-\sigma_{i}(s)\right), \\
i=1,2, \ldots, n .
\end{array}
$$

It is clear that in order to prove that systems (4) and (5) have at least one $\omega$-periodic solution, we only need to prove that system (18) has at least one $\omega$-periodic solution.

Now, for convenience of statements we denote the functions

$$
a_{i j}(t)=\sum_{l=1}^{m} a_{i j l}(t), \quad i, j=1,2, \ldots, n .
$$

The following theorem is about the existence of positive periodic solutions of system (4). 
Theorem 4. Suppose that assumption (H1) holds and there exists a constant $\theta_{i}>0, \zeta_{i}>0, i=1,2, \ldots, n$, such that

$$
\begin{array}{r}
\min _{t \in[0, \omega]}\left\{\sum_{l=1}^{m}\left[\delta_{i i l}(t) \theta_{i}-\sum_{j \neq i}^{n} \delta_{j i l}(t) \theta_{j}\right]\right\} \\
=: \zeta_{i}>0, \\
i=1,2, \ldots, n,
\end{array}
$$

where

$$
\begin{gathered}
\delta_{i j l}(t)=\frac{a_{i j l}\left(\varphi_{i j l}(t)\right)}{1-\tau_{i j l}^{\prime}\left(\varphi_{i j l}(t)\right)}, \\
i, j=1,2, \ldots, n, l=1,2, \ldots, m,
\end{gathered}
$$

and the algebraic equation

$$
\bar{r}_{i}-\bar{H}_{i}-\bar{a}_{i i} v_{i}+\sum_{j \neq i}^{n} \bar{a}_{i j} v_{j}=0, \quad i=1,2, \ldots, n,
$$

where

$$
\begin{aligned}
& H_{i}=\int_{0}^{\omega}\left(\beta_{i}(s)+\gamma_{i}(s)\right) \\
& \times\left(d_{i}(t) G_{i}(t, s)+e_{i}(t) G_{i}\left(t-\varepsilon_{i}(t), s\right)\right) d s, \\
& \quad i=1,2, \ldots, n,
\end{aligned}
$$

has a unique positive solution. Then system (4) has at least one positive $\omega$-periodic solution.

Proof. For system (18) we introduce new variables $y_{i}(t)(i=$ $1,2, \ldots, n)$ such that

$$
x_{i}(t)=\exp \left\{y_{i}(t)\right\}, \quad i=1,2, \ldots, n .
$$

Then system (18) is rewritten in the following form:

$$
\begin{aligned}
\dot{y}_{i}(t)= & r_{i}(t)-\sum_{j=1}^{2}(-1)^{i+j} \sum_{l=1}^{m} a_{i j l}(t) \exp \left\{y_{j}\left(t-\tau_{i j l}(t)\right)\right\} \\
& -\exp \left\{-y_{i}(t)\right\} d_{i}(t) U_{i}(t), \\
& -\exp \left\{-y_{i}(t)\right\} e_{i}(t) U_{i}\left(t-\varepsilon_{i}(t)\right) \\
U_{i}(t)= & \int_{t}^{t+\omega} K\left(e^{y_{i}}\right) G_{i}(t, s) d s, \quad i=1,2,
\end{aligned}
$$

where

$$
\begin{array}{r}
K\left(e^{y_{i}}\right)=\beta_{i}(s) \exp \left\{y_{i}(s)\right\}+\gamma_{i}(s) \exp \left\{y_{i}\left(s-\sigma_{i}(s)\right)\right\}, \\
i=1,2 .
\end{array}
$$

In order to apply Lemma 2 to system (26), we introduce the normed vector spaces $X$ and $Z$ as follows. Let
$C\left(R, R^{n}\right)$ denote the space of all continuous functions $y(t)=$ $\left(y_{1}(t), y_{2}(t), \ldots, y_{n}(t)\right): R \rightarrow R^{n}$. We take

$$
\begin{aligned}
X & =Z \\
& =\left\{y(t) \in C\left(R, R^{n}\right): y(t) \text { is an } \omega \text {-periodic function }\right\},
\end{aligned}
$$

with norm

$$
\|y\|=\sum_{i=1}^{n} \max _{t \in[0, \omega]}\left|y_{i}(t)\right| .
$$

It is obvious that $X$ and $Z$ are the Banach spaces. We define a linear operator $L: \operatorname{Dom} L \subset X \rightarrow Z$ and a continuous operator $N: X \rightarrow Z$ as follows:

$$
\begin{gathered}
L y(t)=\dot{y}(t), \\
N y(t)=\left(N y_{1}(t), N y_{2}(t), \ldots, N y_{n}(t)\right),
\end{gathered}
$$

where

$$
\begin{aligned}
N y_{i}(t)= & r_{i}(t)-\sum_{l=1}^{m} a_{i i l}(t) \exp \left\{y_{i}\left(t-\tau_{i i l}(t)\right)\right\} \\
& +\sum_{j \neq i}^{n} \sum_{l=1}^{m} a_{i j l}(t) \exp \left\{y_{j}\left(t-\tau_{i j l}(t)\right)\right\} \\
& -\exp \left\{-y_{i}(t)\right\} d_{i}(t) u_{i}(t)-\exp \left\{-y_{i}(t)\right\} \\
& \times e_{i}(t) u_{i}\left(t-\varepsilon_{i}(t)\right) .
\end{aligned}
$$

Further, we define continuous projectors $P: X \rightarrow X$ and $Q: Z \rightarrow Z$ as follows:

$$
P y(t)=\frac{1}{\omega} \int_{0}^{\omega} y(t) d t, \quad Q v(t)=\frac{1}{\omega} \int_{0}^{\omega} v(t) d t .
$$

We easily see $\operatorname{Im} L=\left\{v \in Z: \int_{0}^{\omega} v(t) d t=0\right\}$ and $\operatorname{Ker} L=R^{n}$. It is obvious that $\operatorname{Im} L$ is closed in $Z$ and $\operatorname{dimKer} L=n$. Since for any $v \in Z$ there are unique $v_{1} \in R^{n}$ and $v_{2} \in \operatorname{Im} L$ with

$$
v_{1}=\frac{1}{\omega} \int_{0}^{\omega} v(t) d t, \quad v_{2}(t)=v(t)-v_{1},
$$

such that $v(t)=v_{1}+v_{2}(t)$, we have codim Im $L=n$. Therefore, $L$ is a Fredholm mapping of index zero. Furthermore, the generalized inverse (to $L) K_{p}: \operatorname{Im} L \rightarrow \operatorname{Ker} P \cap \operatorname{Dom} L$ is given in the following form:

$$
K_{p} v(t)=\int_{0}^{t} v(s) d s-\frac{1}{\omega} \int_{0}^{\omega} \int_{0}^{t} v(s) d s d t
$$

For convenience, we denote $F(t)=\left(F_{1}(t), F_{2}(t), \ldots, F_{n}(t)\right)$ as follows:

$$
\begin{aligned}
F_{i}(t)= & r_{i}(t)-\sum_{l=1}^{m} a_{i i l}(t) \exp \left\{y_{i}\left(t-\tau_{i i l}(t)\right)\right\} \\
& +\sum_{j \neq i}^{n} \sum_{l=1}^{m} a_{i j l}(t) \exp \left\{y_{j}\left(t-\tau_{i j l}(t)\right)\right\} \\
& -\exp \left\{-y_{i}(t)\right\} d_{i}(t) u_{i}(t)-\exp \left\{-y_{i}(t)\right\} \\
& \times e_{i}(t) u_{i}\left(t-\varepsilon_{i}(t)\right) .
\end{aligned}
$$


Thus, we have

$$
\begin{gathered}
\text { QNy }(t)=\frac{1}{\omega} \int_{0}^{\omega} F(t) d t \\
K_{p}(I-Q) N u(t)=K_{p} I N u(t)-K_{p} Q N u(t) \\
=\int_{0}^{t} F(s) d s-\frac{1}{\omega} \int_{0}^{\omega} \int_{0}^{t} F(s) d s d t \\
+\left(\frac{1}{2}-\frac{t}{\omega}\right) \int_{0}^{\omega} F(s) d s .
\end{gathered}
$$

From formulas (36), we easily see that $Q N$ and $K_{p}(I-Q) N$ are continuous operators. Furthermore, it can be verified that $\overline{K_{p}(I-Q) N(\bar{\Omega})}$ is compact for any open bounded set $\Omega \subset X$ by using the Arzela-Ascoli theorem and $Q N(\bar{\Omega})$ is bounded. Therefore, $N$ is $L$-compact on $\bar{\Omega}$ for any open bounded subset $\Omega \subset X$.

Now, we reach the position to search for an appropriate open bounded subset $\Omega$ for the application of the continuation theorem (Lemma 2) to system (26).

Corresponding to the operator equation $L y(t)=\lambda N y(t)$ with parameter $\lambda \in(0,1)$, we have

$$
\dot{y}_{i}(t)=\lambda F_{i}(t), \quad i=1,2, \ldots, n,
$$

where $F_{i}(t)(i=1,2, \ldots, n)$ are given in (35).

Assume that $y(t)=\left(y_{1}(t), y_{2}(t), \ldots, y_{n}(t)\right) \in X$ is a solution of system (37) for some parameter $\lambda \in(0,1)$. By integrating system $(37)$ over the interval $[0, \omega]$, we obtain

$$
\begin{aligned}
& \int_{0}^{\omega}\left[r_{i}(t)-\sum_{l=1}^{m} a_{i i l}(t) \exp \left\{y_{i}\left(t-\tau_{i i l}(t)\right)\right\}\right. \\
& \quad+\sum_{j \neq i l=1}^{n} \sum_{i j l}^{m} a_{i j}(t) \exp \left\{y_{j}\left(t-\tau_{i j l}(t)\right)\right\} \\
& \quad-e_{i}(t) \exp \left\{-y_{i}(t)\right\} \int_{t-\varepsilon_{i}(t)}^{t-\varepsilon_{i}(t)+\omega} K\left(e^{y_{i}}\right) G_{i}\left(t-\varepsilon_{i}(t), s\right) d s \\
& \left.\quad-d_{i}(t) \exp \left\{-y_{i}(t)\right\} \int_{t}^{t+\omega} K\left(e^{y_{i}}\right) G_{i}(t, s) d s\right] d t=0, \\
& i=1,2, \ldots, n .
\end{aligned}
$$

Consequently,

$$
\begin{aligned}
\bar{r}_{i} \omega & +\int_{0}^{\omega}\left[\sum_{j \neq i}^{n} \sum_{l=1}^{m} a_{i j l}(t) \exp \left\{y_{j}\left(t-\tau_{i j l}(t)\right)\right\}\right] d t \\
= & \int_{0}^{\omega}\left[\sum_{l=1}^{m} a_{i i l}(t) \exp \left\{y_{i}\left(t-\tau_{i i l}(t)\right)\right\}\right] d t \\
& +\int_{0}^{\omega} e_{i}(t) \exp \left\{-y_{i}(t)\right\}
\end{aligned}
$$

$$
\begin{array}{r}
\times \int_{t-\varepsilon_{i}(t)}^{t-\varepsilon_{i}(t)+\omega} K\left(e^{y_{i}}\right) G_{i}\left(t-\varepsilon_{i}(t), s\right) d s d t \\
+\int_{0}^{\omega} d_{i}(t) \exp \left\{-y_{i}(t)\right\} \int_{t}^{t+\omega} K\left(e^{y_{i}}\right) G_{i}(t, s) d s d t \\
i=1,2, \ldots, n .
\end{array}
$$

From the continuity of $y(t)=\left(y_{1}(t), y_{2}(t), \ldots, y_{n}(t)\right)$, there exist constants $\xi_{i}, \eta_{i} \in[0, \omega](i=1,2, \ldots, n)$ such that

$$
\begin{aligned}
y_{i}\left(\xi_{i}\right)=\max _{t \in[0, \omega]} y_{i}(t), \quad y_{i}\left(\eta_{i}\right) & =\min _{t \in[0, \omega]} y_{i}(t) \\
i & =1,2, \ldots, n .
\end{aligned}
$$

By (39) and (40) we obtain

$$
\begin{aligned}
\bar{r}_{i} \omega \leq & \int_{0}^{\omega} a_{i i}(t) \exp \left\{y_{i}\left(\xi_{i}\right)\right\} d t \\
& +\int_{0}^{\omega} A_{i}(t) \exp \left\{y_{i}\left(\xi_{i}\right)\right\} d t, \quad i=1,2, \ldots, n,
\end{aligned}
$$

where

$$
\begin{gathered}
A_{i}(t)=e_{i}(t) \int_{t-\varepsilon_{i}(t)}^{t-\varepsilon_{i}(t)+\omega} G_{i}\left(t-\varepsilon_{i}(t), s\right)\left(\beta_{i}(s)+\gamma_{i}(s)\right) d s \\
+d_{i}(t) \int_{t}^{t+\omega} G_{i}(t, s)\left(\beta_{i}(s)+\gamma_{i}(s)\right) d s, \\
i=1,2, \ldots, n .
\end{gathered}
$$

Therefore, we further have

$$
y_{i}\left(\xi_{i}\right) \geq \ln \left(\frac{\bar{r}_{i}}{\bar{a}_{i i}+\bar{A}_{i}}\right), \quad i=1,2, \ldots, n .
$$

Let $s_{i j l}(t)=t-\tau_{i j l}(t)(i, j=1,2, \ldots, n, l=1,2, \ldots, m)$; then from Lemma 1 and (H1) we get that function $s_{i j l}(t)$ has a unique $\omega$ periodic inverse function $\varphi_{i j l}(t)$; then, for every $i, j=1,2, \ldots, n, l=1,2, \ldots, m$, we have

$$
\begin{aligned}
\int_{0}^{\omega} a_{i j l}(t) \exp \left\{y_{i}\left(t-\tau_{i j l}(t)\right)\right\} d t \\
\quad=\int_{-\tau_{i j l}(0)}^{\omega-\tau_{i j l}(\omega)} \frac{a_{i j l}\left(\varphi_{i j l}(t)\right)}{1-\tau_{i j l}^{\prime}\left(\varphi_{i j l}(t)\right)} \exp \left\{y_{i}(t)\right\} d t .
\end{aligned}
$$

One can see that

$$
\begin{gathered}
\frac{a_{i j l}\left(\varphi_{i j l}(t)\right)}{1-\tau_{i j l}^{\prime}\left(\varphi_{i j l}(t)\right)}=: \delta_{i j l}(t), \\
i, j=1,2, \ldots, n, l=1,2, \ldots, m,
\end{gathered}
$$

are $\omega$ periodic functions. Then, for every $i, j=1,2, \ldots, n, l=$ $1,2, \ldots, m$, we have

$$
\int_{0}^{\omega} a_{i j l}(t) \exp \left\{y_{i}\left(t-\tau_{i j l}(t)\right)\right\} d t=\int_{0}^{\omega} \delta_{i j l}(t) \exp \left\{y_{i}(t)\right\} d t .
$$


From (39) and (46) we further obtain

$$
\begin{aligned}
& \sum_{i=1}^{n}\left(\int _ { 0 } ^ { \omega } \left(\sum_{l=1}^{m} \delta_{i i l}(t) \exp \left\{y_{i}(t)\right\}\right.\right.+d_{i}(t) \exp \left\{-y_{i}(t)\right\} \int_{t}^{t+\omega} K\left(e^{y_{i}}\right) G_{i}(t, s) d s \\
&+e_{i}(t) \exp \left\{-y_{i}(t)\right\} \\
&\left.\left.\times \int_{t-\varepsilon_{i}(t)}^{t-\varepsilon_{i}(t)+\omega} K\left(e^{y_{i}}\right) G_{i}\left(t-\varepsilon_{i}(t), s\right) d s\right) d t\right) \\
&=\sum_{i=1}^{n}\left(\bar{r}_{i} \omega+\int_{0}^{\omega}\left[\sum_{j \neq i}^{n} \sum_{l=1}^{m} \delta_{i j l}(t) \exp \left\{y_{j}(t)\right\}\right] d t\right) .
\end{aligned}
$$

From the above equality we have

$$
\begin{aligned}
& \sum_{i=1}^{n}\left(\int_{0}^{\omega}\left[\sum_{l=1}^{m} \delta_{i i l}(t)-\sum_{j \neq i}^{n} \sum_{l=1}^{m} \delta_{j i l}(t)\right] \exp \left\{y_{i}(t)\right\} d t\right) \\
& \leq \sum_{i=1}^{n} \bar{r}_{i} \omega, \\
& \int_{0}^{\omega}\left[\sum_{l=1}^{m} \delta_{i i l}(t)-\sum_{j \neq i}^{n} \sum_{l=1}^{m} \delta_{j i l}(t)\right] \exp \left\{y_{i}(t)\right\} d t \\
& \leq \sum_{i=1}^{n} \bar{r}_{i} \omega, \quad i=1,2, \ldots, n .
\end{aligned}
$$

From the assumptions of Theorem 4 and (48) we can obtain

$$
\zeta_{i} \int_{0}^{\omega} \exp \left\{y_{i}(t)\right\} d t \leq \sum_{i=1}^{n} \bar{r}_{i} \omega, \quad i=1,2, \ldots, n
$$

Consequently,

$$
\int_{0}^{\omega} \exp \left\{y_{i}(t)\right\} d t \leq \frac{\sum_{i=1}^{n} \bar{r}_{i} \omega}{\zeta_{i}}, \quad i=1,2, \ldots, n
$$

From (40) and (50), we further obtain

$$
y_{i}\left(\eta_{i}\right) \leq \ln \left(\frac{\sum_{i=1}^{n} \bar{r}_{i}}{\zeta_{i}}\right), \quad i=1,2, \ldots, n
$$

On the other hand, directly from system (26) we have

$$
\begin{aligned}
& \int_{0}^{\omega}\left|\dot{y}_{i}(t)\right| d t \\
& \leq \int_{0}^{\omega}\left|r_{i}(t)\right| d t \\
& \quad+\int_{0}^{\omega}\left(\sum_{l=1}^{m} a_{i i l}(t) \exp \left\{y_{i}\left(t-\tau_{i i l}(t)\right)\right\}\right. \\
& \leq \int_{0}^{\omega}\left|r_{i}(t)\right| d t \\
& \left.\quad+\int_{j \neq i}^{n} \sum_{l=1}^{m} a_{i j l}(t) \exp \left\{y_{j}\left(t-\tau_{i j l}(t)\right)\right\}\right) d t \\
& \leq \int_{l=1}^{\omega}\left|\delta_{i}(t)\right| d t+D_{i}^{M} \int_{0}^{\omega} \exp \left\{y_{i}(t)\right\} d t \\
& \leq\left|\bar{r}_{i}\right| \omega+\frac{D_{i}^{M} \sum_{i=1}^{n} \bar{r}_{i} \omega}{\zeta_{i}}, i=1,2, \ldots, n,
\end{aligned}
$$

where

$$
D_{i}(t)=\sum_{l=1}^{m}\left(\delta_{i i l}(t)+\sum_{j \neq i}^{n} \delta_{j i l}(t)\right), \quad i=1,2, \ldots, n
$$

From (43), (51), and (52), we have for any $t \in[0, \omega]$

$$
\begin{aligned}
& y_{i}(t) \leq y_{i}\left(\eta_{i}\right)+\int_{0}^{\omega}\left|\dot{y}_{i}(t)\right| d t \\
& \leq \ln \left(\frac{\sum_{i=1}^{n} \bar{r}_{i}}{\zeta_{i}}\right)+\left|\bar{r}_{i}\right| \omega+\frac{D_{i}^{M} \sum_{i=1}^{n} \bar{r}_{i} \omega}{\zeta_{i}}=: M_{i}, \quad(54) \\
& i=1,2, \ldots, n, \\
& y_{i}(t) \geq y_{i}\left(\xi_{i}\right)-\int_{0}^{\omega}\left|\dot{y}_{i}(t)\right| d t \\
& \geq \ln \left(\frac{\bar{r}_{i}}{\bar{a}_{i i}+\bar{A}_{i}}\right)-\left|\bar{r}_{i}\right| \omega-\frac{D_{i}^{M} \sum_{i=1}^{n} \bar{r}_{i} \omega}{\zeta_{i}}=: N_{i}, \\
& i=1,2, \ldots, n
\end{aligned}
$$

Therefore, from (54) and (55), we have

$$
\max _{t \in[0, \omega]}\left|y_{i}(t)\right| \leq \max \left\{\left|M_{i}\right|,\left|N_{i}\right|\right\}=: B_{i}, \quad i=1,2, \ldots, n .
$$


It can be seen that the constants $B_{i}(i=1,2, \ldots, n)$ are independent of parameter $\lambda \in(0,1)$. For any $y=$ $\left(y_{1}, y_{2}, \ldots, y_{n}\right) \in R^{n}$, from (31) we can obtain

$$
\begin{gathered}
\text { QNy }=\left(Q N y_{1}, Q N y_{2}, \ldots, Q N y_{n}\right) \\
Q N y_{i}=\bar{r}_{i}-\bar{H}_{i}-\bar{a}_{i i} \exp \left\{y_{i}\right\}+\sum_{j \neq i}^{n} \bar{a}_{i j} \exp \left\{y_{j}\right\}, \\
i=1,2, \ldots, n, \\
H_{i}(t)=\int_{0}^{\omega}\left(\beta_{i}(s)+\gamma_{i}(s)\right)\left(d_{i}(t) G_{i}(t, s)\right) \\
+e_{i}(t) G_{i}\left(t-\varepsilon_{i}(t), s\right) d s, \quad i=1,2, \ldots, n .
\end{gathered}
$$

We consider the following algebraic equation:

$$
\bar{r}_{i}-\bar{H}_{i}-\bar{a}_{i i} v_{i}+\sum_{j \neq i}^{n} \bar{a}_{i j} v_{j}=0, \quad i=1,2, \ldots, n .
$$

From the assumption of Theorem 4 , the equation has a unique positive solution $v^{*}=\left(v_{1}^{*}, v_{2}^{*}, \ldots, v_{n}^{*}\right)$. Hence, the equation $\mathrm{QNy}=0$ has a unique solution $y^{*}=$ $\left(y_{1}^{*}, y_{2}^{*}, \ldots, y_{n}^{*}\right)=\left(\ln v_{1}^{*}, \ln v_{2}^{*}, \ldots, \ln v_{n}^{*}\right) \in R^{n}$.

Choosing constant $B>0$ large enough such that $\left|y_{1}^{*}\right|+$ $\left|y_{2}^{*}\right|+\cdots+\left|y_{n}^{*}\right|<B$ and $B>B_{1}+B_{2}+\cdots+B_{n}$, we define a bounded open set $\Omega \subset X$ as follows:

$$
\Omega=\{y \in X:\|y\|<B\} .
$$

It is clear that $\Omega$ satisfies conditions $(a)$ and $(b)$ of Lemma 2. On the other hand, by directly calculating we can obtain

$$
\begin{aligned}
\operatorname{deg}\{J Q N, & \Omega \cap \operatorname{Ker} L,(0,0, \ldots, 0)\} \\
= & \operatorname{sgn}\left|\begin{array}{cccc}
f_{y_{1}}^{1} & f_{y_{2}}^{1} & \cdots & f_{y_{n}}^{1} \\
f_{y_{1}}^{2} & f_{y_{2}}^{2} & \cdots & f_{y_{n}}^{2} \\
\cdots & \cdots & \cdots & \cdots \\
f_{y_{1}}^{n} & f_{y_{2}}^{n} & \cdots & f_{y_{n}}^{n}
\end{array}\right|,
\end{aligned}
$$

where

$$
\begin{gathered}
f_{y_{j}}^{i}=-\bar{a}_{i j} \exp \left\{y_{j}^{*}\right\}, \quad i=j \\
f_{y_{j}}^{i}=\bar{a}_{i j} \exp \left\{y_{j}^{*}\right\}, \quad i \neq j i, j=1,2, \ldots, n .
\end{gathered}
$$

From the assumption of Theorem 4, we have

$$
\left|\begin{array}{cccc}
f_{y_{1}}^{1} & f_{y_{2}}^{1} & \cdots & f_{y_{n}}^{1} \\
f_{y_{1}}^{2} & f_{y_{2}}^{2} & \cdots & f_{y_{n}}^{2} \\
\cdots & \cdots & \cdots & \cdots \\
f_{y_{1}}^{n} & f_{y_{2}}^{n} & \cdots & f_{y_{n}}^{n}
\end{array}\right| \neq 0 .
$$

From this, we finally have

$$
\operatorname{deg}\{J Q N, \Omega \cap \operatorname{Ker} L,(0,0, \ldots, 0)\} \neq 0 .
$$

This shows that $\Omega$ satisfies condition (c) of Lemma 2 . Therefore, system (26) has an $\omega$-periodic solution $y^{*}(t)=$ $\left(y_{1}^{*}(t), y_{2}^{*}(t), \ldots, y_{n}^{*}(t)\right) \in \bar{\Omega}$. Finally, we have system that (4) has a positive $\omega$-periodic solution. This completes the proof.

\section{Acknowledgments}

This work was supported by the Natural Science Foundation of Xinjiang (Grants no. 2012223177, 200721104) and the National Natural Science Foundation of China (Grants no. 10661010, 11261061).

\section{References}

[1] X. Song and L. Chen, "Persistence and global stability for nonautonomous predator-prey system with diffusion and time delay," Computers and Mathematics with Applications, vol. 35, no. 6, pp. 33-40, 1998.

[2] Y. Pinghua and X. Rui, "Global attractivity of the periodic Lotka-Volterra system," Journal of Mathematical Analysis and Applications, vol. 233, no. 1, pp. 221-232, 1999.

[3] F. Wei, Y. Lin, L. Que, Y. Chen, Y. Wu, and Y. Xue, "Periodic solution and global stability for a nonautonomous competitive Lotka-Volterra diffusion system," Applied Mathematics and Computation, vol. 216, no. 10, pp. 3097-3104, 2010.

[4] R. Tan, Z. Liu, and R. A. Cheke, "Periodicity and stability in a single-species model governed by impulsive differential equation," Applied Mathematical Modelling, vol. 36, no. 3, pp. 1085-1094, 2012.

[5] K. Deimling, Nonlinear Functional Analysis, Springer, Beijing, China, 1988.

[6] Y. Xiao, W. Wang, and J. Chen, "Permanence and periodic solution of nonautonomous delay Lotka-Volterra diffusion system," Systems Science and Mathematical Sciences, vol. 12, no. 4, pp. 344-349, 1999.

[7] T. Zhidong, "The almost periodic Kolmogorov competitive systems," Nonlinear Analysis: Theory, Methods and Applications, vol. 42, no. 7, pp. 1221-1230, 2000.

[8] F. Chen, "On the periodic solutions of periodic multi-species Kolmogorov type competitive system with delays and feedback controls," Applied Mathematics and Computation, vol. 180, no. 1, pp. 366-373, 2006.

[9] K. Deimling, Nonlinear Functional Analysis, Springer, New York, NY, USA, 1985.

[10] M. A. Krasnoselskii, Positive Solutions of Operator Equations, P. Noordhoff, Groningen, The Netherlands, 1964.

[11] D. J. Guo and V. Lakshmikantham, Nonlinear Problems in Abstract Cones, vol. 5, Academic Press, New York, NY, USA, 1988.

[12] J. Yan, "Global positive periodic solutions of periodic $n$-species competition systems," Journal of Mathematical Analysis and Applications, vol. 356, no. 1, pp. 288-294, 2009.

[13] W. Yan and J. Yan, "Periodicity and asymptotic stability of a predator-prey system with infinite delays," Computers and Mathematics with Applications, vol. 60, no. 5, pp. 1465-1472, 2010.

[14] J. Yan and G. Liu, "Periodicity and stability for a LotkaVolterra type competition system with feedback controls and deviating arguments," Nonlinear Analysis: Theory, Methods and Applications, vol. 74, no. 9, pp. 2916-2928, 2011.

[15] W. A. Horn, "Some fixed point theorems for compact maps and flows in Banach spaces," Transactions of the American Mathematical Society, vol. 149, pp. 391-404, 1970.

[16] Z. D. Teng and L. S. Chen, "The positive periodic solutions of periodic Kolmogorov type systems with delays," Acta Mathematicae Applicatae Sinica, vol. 22, no. 3, pp. 446-456, 1999 (Chinese). 
[17] H.-F. Huo and W.-T. Li, "Positive periodic solutions of a class of delay differential system with feedback control," Applied Mathematics and Computation, vol. 148, no. 1, pp. 35-46, 2004.

[18] P. Weng, "Existence and global stability of positive periodic solution of periodic integrodifferential systems with feedback controls," Computers and Mathematics with Applications, vol. 40, no. 6-7, pp. 747-759, 2000.

[19] F. Yang and D. Jiang, "Existence and global attractivity of positive periodic solution of a logistic growth system with feedback control and deviating arguments," Annals of Differential Equations, vol. 17, no. 4, pp. 377-384, 2001.

[20] Y. Li, P. Liu, and L. Zhu, "Positive periodic solutions of a class of functional differential systems with feedback controls," Nonlinear Analysis: Theory, Methods and Applications, vol. 57, no. 5-6, pp. 655-666, 2004.

[21] F. Chen, "Positive periodic solutions of neutral Lotka-Volterra system with feedback control," Applied Mathematics and Computation, vol. 162, no. 3, pp. 1279-1302, 2005.

[22] D. Hu and Z. Zhang, "Four positive periodic solutions to a Lotka-Volterra cooperative system with harvesting terms," Nonlinear Analysis: Real World Applications, vol. 11, no. 2, pp. 1115-1121, 2010.

[23] K. Zhao and Y. Ye, "Four positive periodic solutions to a periodic Lotka-Volterra predatory-prey system with harvesting terms," Nonlinear Analysis: Real World Applications, vol. 11, no. 4, pp. 2448-2455, 2010.

[24] H.-F. Huo and W.-T. Li, "Positive periodic solutions of a class of delay differential system with feedback control," Applied Mathematics and Computation, vol. 148, no. 1, pp. 35-46, 2004.

[25] H. Fang and Y. Xiao, "Existence of multiple periodic solutions for delay Lotka-Volterra competition patch systems with harvesting," Applied Mathematical Modelling, vol. 33, no. 2, pp. 1086-1096, 2009.

[26] G. Lin and Y. Hong, "Periodic solutions to non autonomous predator prey system with delays," Nonlinear Analysis: Real World Applications, vol. 10, no. 3, pp. 1589-1600, 2009.

[27] S. Lu, "On the existence of positive periodic solutions to a Lotka Volterra cooperative population model with multiple delays," Nonlinear Analysis: Theory, Methods and Applications, vol. 68, no. 6, pp. 1746-1753, 2008.

[28] J. Shen and J. Li, "Existence and global attractivity of positive periodic solutions for impulsive predator-prey model with dispersion and time delays," Nonlinear Analysis: Real World Applications, vol. 10, no. 1, pp. 227-243, 2009.

[29] F. Yin and Y. Li, "Positive periodic solutions of a single species model with feedback regulation and distributed time delay," Applied Mathematics and Computation, vol. 153, no. 2, pp. 475484, 2004.

[30] A. Muhammadhaji and Z. Teng, "Positive periodic solutions of n-species Lotka-Volterra cooperative systems with delays," Vietnam Journal of Mathematics, vol. 40, no. 4, pp. 453-467, 2012.

[31] A. Muhammadhaji and Z. Teng, "Global attractivity of a periodic delayed $\mathrm{N}$-species model of facultative mutualism," Discrete Dynamics in Nature and Society, vol. 2013, Article ID 580185, 11 pages, 2013.

[32] M. Kouche, N. Tatar, and S. Liu, "Permanence and existence of a positive periodic solution to a periodic stage-structured system with infinite delay," Applied Mathematics and Computation, vol. 202, no. 2, pp. 620-638, 2008.
[33] L. Zhang, H.-X. Li, and X.-B. Zhang, "Periodic solutions of competition Lotka-Volterra dynamic system on time scales," Computers and Mathematics with Applications, vol. 57, no. 7, pp. 1204-1211, 2009.

[34] S. Lu, "On the existence of positive periodic solutions for neutral functional differential equation with multiple deviating arguments," Journal of Mathematical Analysis and Applications, vol. 280, no. 2, pp. 321-333, 2003.

[35] Z. Zhang, J. Wu, and Z. Wang, "Periodic solutions of nonautonomous stage-structured cooperative system," Computers and Mathematics with Applications, vol. 47, no. 4-5, pp. 699-706, 2004.

[36] R. E. Gaines and J. L. Mawhin, Coincidence Degree, and Nonlinear Differential Equations, vol. 568 of Lecture Notes in Mathematics, Springer, Berlin, Germany, 1977. 


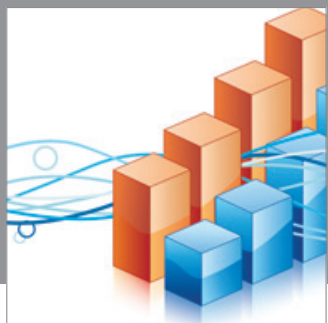

Advances in

Operations Research

mansans

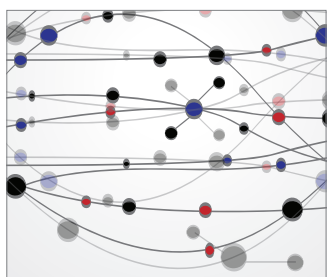

The Scientific World Journal
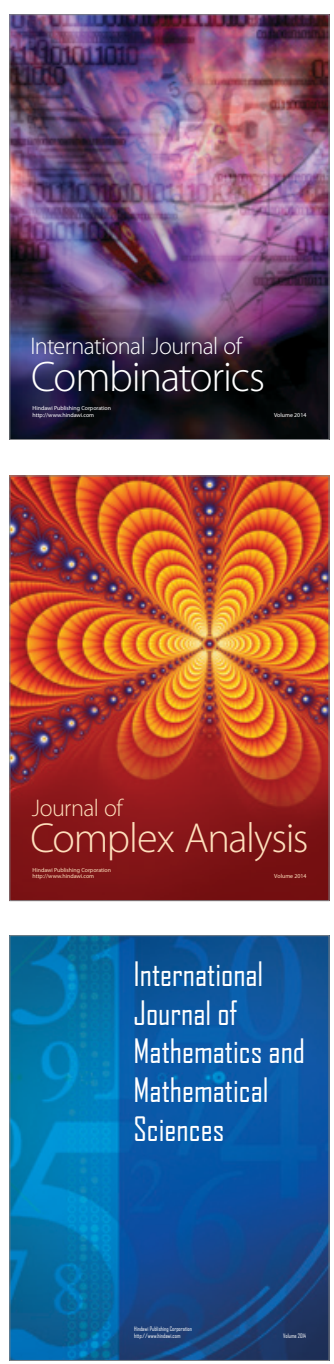
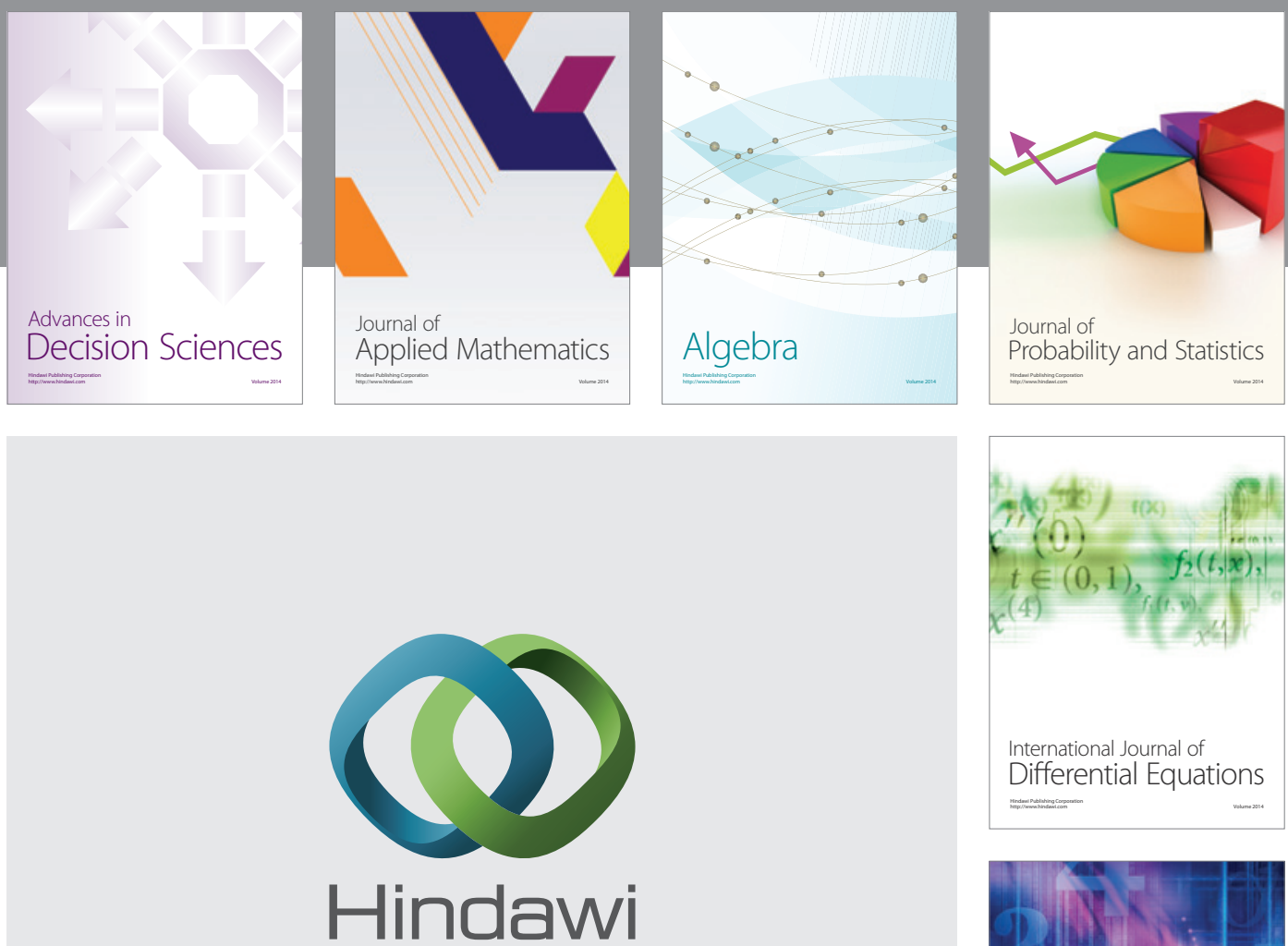

Submit your manuscripts at http://www.hindawi.com
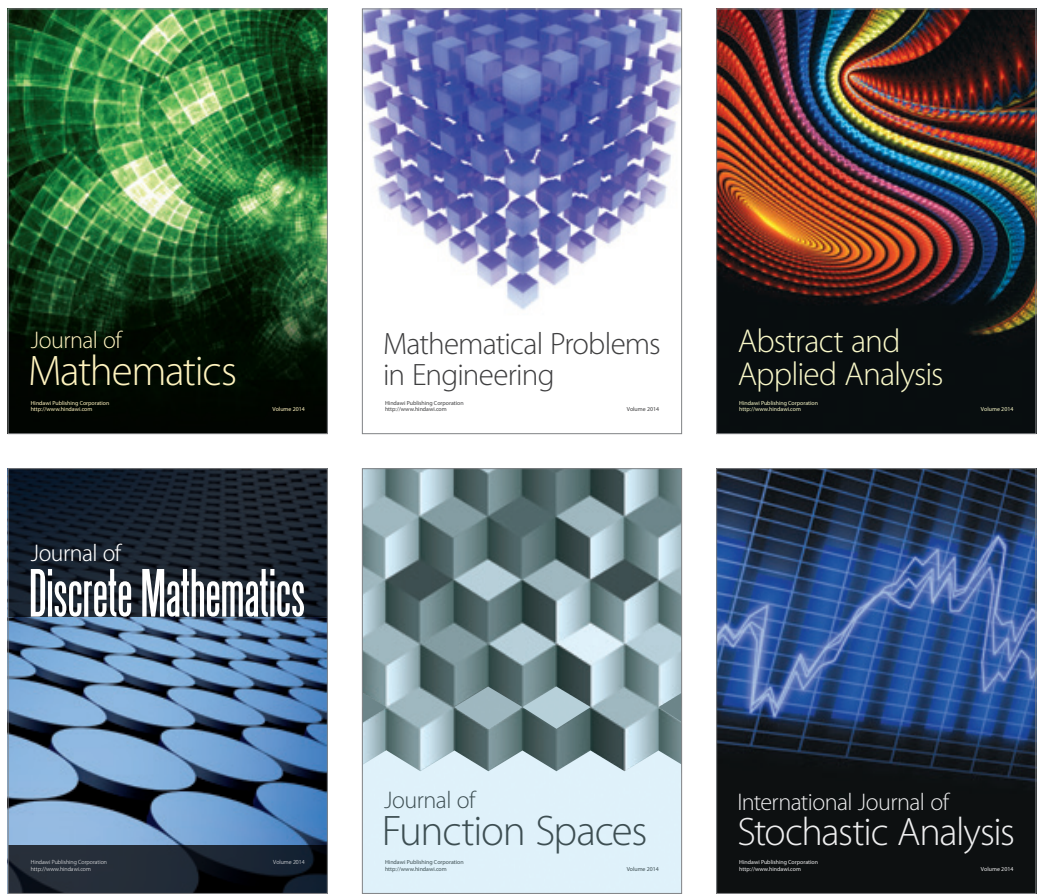

Journal of

Function Spaces

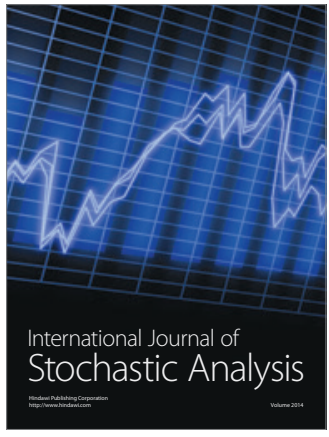

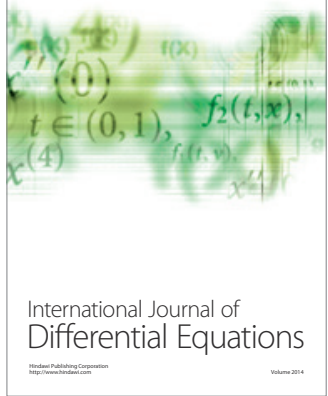
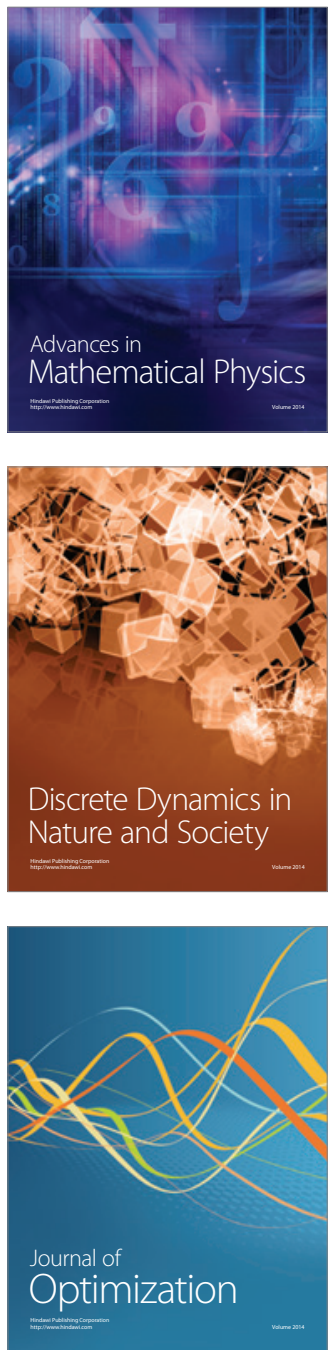